\title{
Variational bound on energy dissipation in plane Couette flow
}

\author{
Rolf Nicodemus*, Siegfried Grossmann ${ }^{\dagger}$, and Martin Holthaus ${ }^{\ddagger}$ \\ Fachbereich Physik der Philipps-Universität, \\ Renthof 6, D-35032 Marburg, Germany
}

(September 20, 2018)

\begin{abstract}
We present numerical solutions to the extended Doering-Constantin variational principle for upper bounds on the energy dissipation rate in turbulent plane Couette flow. Using the compound matrix technique in order to reformulate this principle's spectral constraint, we derive a system of equations that is amenable to numerical treatment in the entire range from low to asymptotically high Reynolds numbers. Our variational bound exhibits a minimum at intermediate Reynolds numbers, and reproduces the Busse bound in the asymptotic regime. As a consequence of a bifurcation of the minimizing wavenumbers, there exist two length scales that determine the optimal upper bound: the effective width of the variational profile's boundary segments, and the extension of their flat interior part.
\end{abstract}

*Electronic address: nicodemu@mailer.uni-marburg.de

†Electronic address: grossmann_s@stat.physik.uni-marburg.de

${ }^{\ddagger}$ Electronic address: holthaus@stat.physik.uni-marburg.de 


\section{INTRODUCTION}

Can one derive upper bounds on the rate of energy dissipation in turbulent shear flows that are both mathematically rigorous and physically meaningful, i.e. "sharp"? This question is of enormous conceptual importance in the theory of turbulence, since the energy dissipation rate is the central object in classical turbulence theories [1 [5], and its behavior at high Reynolds numbers may reveal whether there are intermittency corrections to classical scaling [6, ]]. Since full numerical simulations of shear flows with Reynolds numbers of the order of $10^{6}$ or above are out of reach, and will remain so in the foreseeable future, rigorous estimates of the dissipation rate derived directly from the Navier-Stokes equations are one of the few tools left to the theorist for approaching such questions.

The idea of bounding the rate of energy dissipation with the help of rigorous inequalities, rather than trying to solve the equations of motion under plausible, but essentially uncontrolled approximations, saw its first prime in the early seventies, with the formulation and further exploration of the Howard-Busse theory [8]. In particular, Busse [9 11] was able to derive an asymptotic upper bound on energy dissipation in plane Couette flow that is in accordance with classical theories, but lies roughly an order of magnitude above experimentally measured data [12,13].

After this state of affairs had remained unchanged for about 25 years, Doering and Constantin put forward a new variational principle for computing quantities characterizing turbulent flows [14 [16]. Whereas the first applications of this principle to the plane Couette problem [14, 17, [8] did not exhaust the principle and produced bounds on the dissipation rate that could not compete with Busse's, subsequent analysis by Kerswell [19] revealed that the Doering-Constantin principle, after being extended along the lines worked out in Ref. [20], actually reproduces the Busse bound in the asymptotic regime. Kerswell arrived at this conclusion by mapping the extended Doering-Constantin principle to another variational principle that also occurs in Busse's theory, and then applying Busse's method of solution. However, as emphasized by Kerswell himself [19], there is still no mathematical proof that Busse's solution is really extremal, although this is generally believed to be the case. Moreover, the Busse bound pertains to the limit of asymptotically high Reynolds numbers only. Hence, what is needed is a solution to the extended Doering-Constantin principle that (i) fully exhausts this principle, (ii) spans the entire range from low to asymptotically high Reynolds numbers, and (iii) is logically independent from Busse's solution.

In this paper, we present a full-fledged numerical analysis of the improved DoeringConstantin variational principle for the plane Couette flow that meets these requirements. The goal here is not merely to derive the best possible bound, but we also wish to explore the working principles of the new method. Even if statements concerning the existence of nonclassical scaling remain out of reach at present, the potential power of the new variational principle lies in its great conceptual clarity. Once thoroughly understood, it might serve as a germ for the formulation of a still more advanced principle that could actually allow one to attack the scaling questions.

We organize our material as follows: after having stated the variational principle in Sec. II, Sec. III will be devoted to the resolution of the most demanding technical difficulty, the implementation of the principle's spectral constraint. It is this constraint that seems to have hindered previous numerical work [21] to reach the asymptotic regime. Our results 
will then be described in Sec. IV; the final Sec. $\mathrm{V}$ offers a concluding discussion. Some important technical details concerning the derivation of the system of differential equations actually used in the numerical computations can be found in Appendix A, while Appendix B contains a brief, but instructive example that illustrates the key points of our method, and underlines its accuracy, without too much technical burden.

Since the numerical work is fairly involved, it would hardly have been possible to carry through the entire investigation without some analytical guidance. Our guide is the example problem of the Couette flow without spanwise degrees of freedom, referred to as the restricted Couette problem in the following, which has been treated in detail in Ref. [22], and which shows several features that will reappear here in the context of the unrestricted problem.

\section{THE VARIATIONAL PRINCIPLE}

We consider a layer of an incompressible fluid with kinematic viscosity $\nu$ that is confined between two infinitely extended, parallel rigid plates separated by a distance $h$. The lower plate, coinciding with the plane $z=0$ of a Cartesian coordinate system, is fixed, whereas the upper plate at $z=h$ is sheared with constant velocity $U$ in the positive $x$-direction. The dynamics of the fluid's velocity field $\mathbf{u}(\mathbf{x}, t)$ are determined by the equations

$$
\begin{aligned}
\partial_{t} \mathbf{u}+\mathbf{u} \cdot \nabla \mathbf{u}+\nabla p=\nu \Delta \mathbf{u} & \text { (Navier-Stokes equations) } \\
\nabla \cdot \mathbf{u}=0 & \text { (incompressibility) } \\
\mathbf{u}(x, y, 0, t)=\mathbf{0}, \quad \mathbf{u}(x, y, h, t)=U \hat{\mathbf{x}} & \text { (no-slip boundary conditions); }
\end{aligned}
$$

here $p$ denotes the kinematic pressure and $\hat{\mathbf{x}}$ is the unit vector in $x$-direction. In addition, in both $x$ - and $y$-direction periodic boundary conditions (b.c.) are imposed on $\mathbf{u}$ and $p$. The time-averaged rate of dissipated energy per mass is given by

$$
\varepsilon_{T} \equiv \frac{1}{T} \int_{0}^{T} \mathrm{~d} t\left\{\frac{\nu}{\Omega} \int_{\Omega} \mathrm{d}^{3} x\left[\sum_{i, j=x, y, z}\left(\partial_{j} u_{i}\right)^{2}\right]\right\},
$$

where $\Omega$ is the periodicity volume. Our aim is to derive a mathematically rigorous upper bound on the long-time limit $\varepsilon$ of $\varepsilon_{T}$,

$$
\varepsilon \equiv \lim _{T \rightarrow \infty} \varepsilon_{T}
$$

Equivalently, we consider the non-dimensionalized dissipation rate

$$
c_{\varepsilon}(R e) \equiv \frac{\varepsilon}{U^{3} h^{-1}},
$$

where $R e=U h / \nu$ is the Reynolds number. For calculating the bound we will employ the background flow method as recently put forward by Doering and Constantin [14]17], and developed further in Ref. 20].

It is known [14] that the rate of energy dissipated by the laminar flow $\mathbf{u}(\mathbf{x}, t)=(U z / h) \hat{\mathbf{x}}$ provides a rigorous lower bound $\underline{c}_{\varepsilon}$ on $c_{\varepsilon}$ for all $R e$, namely $c_{\varepsilon}(R e) \geq \underline{c_{\varepsilon}}(R e) \equiv R e^{-1}$. Moreover, energy stability theory [23,24] guarantees that the laminar flow is the only possible stable flow at least up to the energy stability limit $R e_{E S}$. Hence, we have the exact identity 


$$
c_{\varepsilon}(R e)=R e^{-1} \quad \text { for } \quad R e<R e_{E S} \approx 82.65
$$

From an early generalization of energy stability theory, the Optimum Theory devised by Busse [9 11], one obtains an approximate asymptotic upper bound [25]:

$$
\lim _{R e \rightarrow \infty} c_{\varepsilon}(R e) \lesssim 0.010
$$

Note that the right hand side of this inequality does not depend on the Reynolds number, so that this bound is in accordance with classical turbulence theories [1] 5]. (For a discussion of the connection between the $R e$-dependence of $c_{\varepsilon}(R e)$ and possible intermittency corrections, see Ref. [6].)

The background flow approach can likewise be considered as a generalization of energy stability theory, but it has a distinctly different twist. Instead of decomposing the velocity field $\mathbf{u}$ into a $z$-dependent, plane-averaged mean flow and the fluctuations around this flow, as Busse did in his Optimum Theory, Doering and Constantin [17] take up an idea by Hopf [26] and write

$$
\mathbf{u}(\mathbf{x}, t)=\mathbf{U}(\mathbf{x})+\mathbf{v}(\mathbf{x}, t) .
$$

Now the stationary and divergence-free auxiliary field $\mathbf{U}(\mathbf{x})$, dubbed the background flow, has to carry the physical b.c., namely $\mathbf{U}(x, y, 0)=\mathbf{0}, \mathbf{U}(x, y, h)=U \hat{\mathbf{x}}$, and $\mathbf{U}(\mathbf{x})$ is periodic in $x$ - and $y$-direction. But apart from these fairly mild specifications, the background flow remains completely arbitrary. Hence, this decomposition (9) serves as the starting point of a variational principle [14]: plug it into the expression (国) for the energy dissipation rate, get rid of the deviations $\mathbf{v}(\mathbf{x}, t)$ with the help of both the equations of motion (11)-(3) and rigorous inequalities that bound the dissipation rate from above, and then adjust the background flow such that this upper bound becomes as low as possible.

For carrying through this program, we restrict ourselves to background flows that are given by a merely height-dependent profile $\phi(\zeta)$, which should reflect the symmetry of the Couette geometry. That is, we admit only background flows of the form

$$
\mathbf{U}(\mathbf{x}) \equiv U \phi(\zeta) \hat{\mathbf{x}}
$$

with

$$
\phi(0)=0, \quad \phi(1)=1, \quad \phi(\zeta)=1-\phi(1-\zeta),
$$

where $\zeta \equiv z / h$ is the dimensionless coordinate in cross-stream direction. The resulting inequality that bounds the dissipation rate then reads 20]

$$
c_{\varepsilon}(R e) \leq\left[1+\frac{a^{2}}{4(a-1)} D\{\phi\}\right] R e^{-1},
$$

where $a>1$ is a dimensionless balance parameter, and $D\{\phi\}$ denotes the profile functional

$$
D\{\phi\} \equiv \int_{0}^{1} \mathrm{~d} \zeta\left[\phi^{\prime}(\zeta)\right]^{2}-1
$$


The bound on the dissipation rate provided by the r.h.s. of the inequality (12) can be minimized by varying both the profile $\phi$ and the balance parameter $a$. However, there is a substantial technical difficulty: the inequality $(19)$ is valid only as long as the profile $\phi$ satisfies a spectral constraint [14]. This spectral constraint demands that all eigenvalues $\lambda$ of the linear eigenvalue problem

$$
\begin{aligned}
\lambda \mathbf{V} & =-2 h^{2} \Delta \mathbf{V}+R \phi^{\prime}\left(\begin{array}{lll}
0 & 0 & 1 \\
0 & 0 & 0 \\
1 & 0 & 0
\end{array}\right) \mathbf{V}+\nabla P, \\
0 & =\nabla \cdot \mathbf{V}, \quad \mathbf{V}(\partial \Omega)=\mathbf{0}
\end{aligned}
$$

for the stationary velocity fields $\mathbf{V}$ be positive. Since the background flow already carries the physical boundary conditions, $\mathbf{V}$ has to satisfy homogeneous b.c., that is, $\mathbf{V}$ has to vanish on the material walls $\partial \Omega$ at $z=0$ and $z=h$, and to be periodic in $x$ - and $y$-direction. Note that the balance parameter $a$ enters into this eigenvalue problem through the rescaled Reynolds number

$$
R \equiv \frac{a}{a-1} R e
$$

In effect, the spectral constraint (14) restricts, for each Reynolds number, the combinations of profiles and balance parameters that are admissible to the variational principle (12). The highly non-trivial task of evaluating this constraint consists in determining, for each candidate profile $\phi$ separately, that rescaled Reynolds number $R$ where the smallest eigenvalue passes through zero. We will denote this number as $R_{c}\{\phi\}$ in the following.

Provided we know how to compute $R_{c}\{\phi\}$ - this task will be taken up in the following section - , each $\phi$ leads to a rigorous upper bound on $c_{\varepsilon}$ in the interval $0 \leq R e<R_{c}\{\phi\}$. After optimization of the balance parameter, this bounds adopts the form [20]

$$
c_{\varepsilon} \leq\left\{\begin{array}{ll}
{[1+D\{\phi\}] R e^{-1}} & \text { for } 0 \leq R e<\frac{1}{2} R_{c}\{\phi\} \\
{\left[1+\frac{D\{\phi\} R_{c}\{\phi\}^{2}}{4\left(R_{c}\{\phi\}-R e\right) R e}\right] R e^{-1}} & \text { for } \frac{1}{2} R_{c}\{\phi\} \leq R e<R_{c}\{\phi\}
\end{array} .\right.
$$

Figure 1 shows the graph of such an upper bound produced by a generic profile $\phi$. When a suitable class of test profiles has been selected, the lower envelope of all graphs stemming from the individual profiles then is the optimal upper bound on $c_{\varepsilon}(R e)$ that can be obtained from this class, i.e., the solution to the variational principle (12) within this class.

\section{EVALUATION OF THE SPECTRAL CONSTRAINT}

We non-dimensionalize the problem by choosing the gapwidth $h$ as the unit of length. By virtue of the linearity of the equations (14), and the homogeneous b.c. imposed on the eigenvectors $\mathbf{V}$, it is not necessary to specify a scale of velocity explicitly. For the sake of notational simplicity we denote all non-dimensionalized quantities by the same symbols as

their dimension-carrying counterparts. For instance, the velocity field $\mathbf{V}(\mathbf{x})$ now is regarded as a dimensionless vector function of the dimensionless coordinates $x, y$ and $z$.

Utilizing the periodic b.c., we start from the ansatz 


$$
\mathbf{V}(\mathbf{x}) \equiv \mathbf{v}(z) \mathrm{e}^{\mathrm{i}\left(k_{x} x+k_{y} y\right)}, \quad P(\mathbf{x}) \equiv p(z) \mathrm{e}^{\mathrm{i}\left(k_{x} x+k_{y} y\right)}
$$

and transform the eigenvalue equations (14) into the system

$$
\begin{aligned}
\lambda v_{x} & =-2\left(\partial_{z}^{2}-k^{2}\right) v_{x}+R \phi^{\prime} v_{z}+\mathrm{i} k_{x} p \\
\lambda v_{y} & =-2\left(\partial_{z}^{2}-k^{2}\right) v_{y}+\mathrm{i} k_{y} p \\
\lambda v_{z} & =-2\left(\partial_{z}^{2}-k^{2}\right) v_{z}+R \phi^{\prime} v_{x}+p^{\prime} \\
0 & =\mathrm{i} k_{x} v_{x}+\mathrm{i} k_{y} v_{y}+v_{z}^{\prime}
\end{aligned}
$$

with

$$
k \equiv \sqrt{k_{x}^{2}+k_{y}^{2}}
$$

The corresponding b.c. for the function $\mathbf{v}(z) \mathrm{read}$

$$
\mathbf{v}(0)=\mathbf{v}(1)=\mathbf{0}
$$

If $k_{y}=0$ we encounter the model problem of a Couette flow without spanwise degrees of freedom. Since this restricted problem has already been studied in detail in Ref. [22], we only need to consider $k_{y} \neq 0$ here.

Defining the linear operator

$$
\mathcal{L} \equiv 2\left(\partial_{z}^{2}-k^{2}\right)+\lambda
$$

Eqs. (17) and (18) together with Eq. (20) yield

$$
p=\frac{1}{k^{2}}\left[\mathcal{L} v_{z}^{\prime}+\mathrm{i} k_{x} R \phi^{\prime} v_{z}\right]
$$

Hence, Eq. (17) can be brought into the form

$$
\mathcal{L} v_{x}=\frac{1}{k^{2}}\left[k_{y}^{2} R \phi^{\prime} v_{z}+\mathrm{i} k_{x} \mathcal{L} v_{z}^{\prime}\right]
$$

and we finally obtain from Eq. (19) a rather intricate equation for $v_{z}(z)$ :

$$
\begin{aligned}
\mathcal{L}^{2}\left(\partial_{z}^{2}-k^{2}\right) v_{z}-4 \frac{\phi^{(2)}}{\phi^{\prime}} \mathcal{L}\left(\partial_{z}^{2}-k^{2}\right) v_{z}^{\prime}-2\left[\frac{\phi^{(3)}}{\phi^{\prime}}-2\left(\frac{\phi^{(2)}}{\phi^{\prime}}\right)^{2}\right] \mathcal{L}\left(\partial_{z}^{2}-k^{2}\right) v_{z} \\
+\left(k_{y} R \phi^{\prime}\right)^{2} v_{z}+\mathrm{i} k_{x} R \phi^{\prime}\left\{2 \mathcal{L} v_{z}^{\prime}+\frac{\phi^{(2)}}{\phi^{\prime}} \mathcal{L} v_{z}+4\left[\frac{\phi^{(3)}}{\phi^{\prime}}-\left(\frac{\phi^{(2)}}{\phi^{\prime}}\right)^{2}\right] v_{z}^{\prime}\right. \\
\left.+2\left[\frac{\phi^{(4)}}{\phi^{\prime}}-3 \frac{\phi^{(3)} \phi^{(2)}}{\left(\phi^{\prime}\right)^{2}}+2\left(\frac{\phi^{(2)}}{\phi^{\prime}}\right)^{3}\right] v_{z}\right\}=0
\end{aligned}
$$

where we rely on the assumption that $\phi^{\prime}(z)$ does not vanish for all $z$ in the interval $[0,1]$. The corresponding b.c. for $v_{z}$ are given by 


$$
\left.v_{z}(z)\right|_{z=0,1}=0,\left.\quad v_{z}^{\prime}(z)\right|_{z=0,1}=0, \quad \text { and }\left.\quad \mathcal{L}\left(\partial_{z}^{2}-k^{2}\right) v_{z}(z)\right|_{z=0,1}=0
$$

Equations (24) and (25) constitute a sixth-order Sturm-Liouville eigenvalue problem. If one succeeds in solving this problem, that is, in computing an eigenvalue $\lambda$ and its eigenfunction $v_{z}^{(\lambda)}(z)$ for some fixed profile $\phi$, wavenumbers $k_{x}$ and $k_{y}$, and a rescaled Reynolds number $R$, then one can determine the associated components $v_{x}^{(\lambda)}(z)$ and $v_{y}^{(\lambda)}(z)$, together with $p^{(\lambda)}(z)$, from Eqs. (23), (18), and (22), so that an eigenvalue and a corresponding eigenvector $\left(\mathbf{V}^{(\lambda)}(\mathbf{x}), P^{(\lambda)}(\mathbf{x})\right)$ of the original eigenvalue problem (14) have been found.

Since we cannot hope to find an analytical solution to this formidable problem for each candidate profile $\phi$, we have to resort to a numerical treatment. This requires to bring the Sturm-Liouville problem into a form that can actually be dealt with on a computer. We defer the technical details of this reformulation, that rely heavily on the so-called compound matrix method [27, to Appendix A, and present only the final equations here. Although these equations may appear, on a cursory glance, even more cumbersome than the original problem posed by Eqs. (24) and (25), they have actually been found well suited for computing the "critical" rescaled Reynolds numbers $R_{c}\{\phi\}$ for all situations of practical interest, as will be amply demonstrated in the following section.

To begin with, we define the abbreviations

$$
R_{x} \equiv \frac{k_{x} R}{k^{2}}, \quad R_{y} \equiv \frac{k_{y} R}{k^{2}}
$$

and the auxiliary functions

$$
\begin{aligned}
\phi_{1}(z) & \equiv \phi^{\prime}(z), \\
\phi_{2}(z) & \equiv \frac{\phi^{(2)}(z)}{2 k}, \\
f_{1}(z) & \equiv 2 \frac{\phi^{(2)}(z)}{\phi^{\prime}(z)} \\
f_{2}(z) & \equiv 3 k+\frac{2}{k} \frac{\phi^{(3)}(z)}{\phi^{\prime}(z)}-\frac{4}{k}\left(\frac{\phi^{(2)}(z)}{\phi^{\prime}(z)}\right)^{2}, \\
g_{1}(z) & \equiv \phi^{\prime}(z)-\frac{1}{k^{2}} \phi^{(3)}(z)+\frac{1}{k^{2}} \frac{\left(\phi^{(2)}(z)\right)^{2}}{\phi^{\prime}(z)}, \\
g_{2}(z) & \equiv \frac{1}{2 k} \phi^{(2)}(z)-\frac{1}{2 k^{3}} \phi^{(4)}+\frac{3}{2 k^{3}} \frac{\phi^{(3)}(z) \phi^{(2)}(z)}{\phi^{\prime}(z)}-\frac{1}{k^{3}} \frac{\left(\phi^{(2)}(z)\right)^{3}}{\left(\phi^{\prime}(z)\right)^{2}}, \\
F_{R_{y}}(z) & =k+\frac{1}{k} \frac{\phi^{(3)}(z)}{\phi^{\prime}(z)}-\frac{2}{k}\left(\frac{\phi^{(2)}(z)}{\phi^{\prime}(z)}\right)^{2}-\frac{1}{4 k}\left(R_{y} \phi^{\prime}(z)\right)^{2} .
\end{aligned}
$$

The compound matrix technique outlined in Appendix A then yields the following system of 20 ordinary, first-order complex differential equations:

$$
y_{1}^{\prime}=k\left[-3 y_{1}+y_{2}-\frac{1}{2} y_{4}-\frac{1}{2} y_{6}\right],
$$




$$
\begin{aligned}
& y_{2}^{\prime}=k\left[-3 y_{2}+y_{3}+y_{5}-\frac{1}{2} y_{8}\right], \\
& y_{3}^{\prime}=k\left[-3 y_{3}+y_{4}+y_{6}\right] \text {, } \\
& y_{4}^{\prime}=k\left[\frac{3}{2} y_{3}-3 y_{4}+y_{7}+\frac{1}{2} y_{10}\right]+f_{1}\left[-2 y_{2}+y_{4}\right]-f_{2} y_{1}-\mathrm{i} R_{x}\left[\phi_{1} y_{2}+\phi_{2}\left(y_{1}+\frac{1}{2} y_{3}\right)\right] \text {, } \\
& y_{5}^{\prime}=k\left[-3 y_{5}+y_{6}+\frac{1}{2} y_{9}+y_{11}\right] \text {, } \\
& y_{6}^{\prime}=k\left[-3 y_{6}+y_{7}+y_{8}+\frac{1}{2} y_{10}+y_{12}\right] \text {, } \\
& y_{7}^{\prime}=k\left[\frac{3}{2} y_{6}-3 y_{7}+y_{9}+y_{13}\right]+f_{1}\left[-y_{1}-2 y_{5}+y_{7}\right]-\mathrm{i} R_{x}\left[\phi_{1} y_{5}+\frac{1}{2} \phi_{2} y_{6}+g_{1} y_{1}\right], \\
& y_{8}^{\prime}=k\left[-3 y_{8}+y_{9}+y_{14}\right] \text {, } \\
& y_{9}^{\prime}=k\left[\frac{3}{2} y_{8}-3 y_{9}+y_{10}+y_{15}\right]+f_{1}\left[-y_{2}+y_{9}\right]+f_{2} y_{5}-\mathrm{i} R_{x}\left[\phi_{2}\left(-y_{5}+\frac{1}{2} y_{8}\right)+g_{1} y_{2}\right] \text {, } \\
& y_{10}^{\prime}=k\left[-3 y_{10}+y_{16}\right]+f_{1}\left[-y_{3}+2 y_{8}+y_{10}\right]+f_{2} y_{6}-\mathrm{i} R_{x}\left[-\phi_{1} y_{8}-\phi_{2} y_{6}+g_{1} y_{3}\right] \text {, } \\
& y_{11}^{\prime}=k\left[-3 y_{11}+y_{12}+\frac{1}{2} y_{15}+\frac{1}{2} y_{17}\right] \text {, } \\
& y_{12}^{\prime}=k\left[-3 y_{12}+y_{13}+y_{14}+\frac{1}{2} y_{16}\right] \text {, } \\
& y_{13}^{\prime}=k\left[\frac{3}{2} y_{12}-3 y_{13}+y_{15}-\frac{1}{2} y_{19}\right]+f_{1}\left[-2 y_{11}+y_{13}\right]+F_{R_{y}} y_{1} \\
& +\mathrm{i} R_{x}\left[-\phi_{1} y_{11}-\frac{1}{2} \phi_{2} y_{12}+g_{2} y_{1}\right] \\
& y_{14}^{\prime}=k\left[-3 y_{14}+y_{15}+y_{17}\right] \text {, } \\
& y_{15}^{\prime}=k\left[\frac{3}{2} y_{14}-3 y_{15}+y_{16}+y_{18}-\frac{1}{2} y_{20}\right]+f_{1} y_{15}+f_{2} y_{11}+F_{R_{y}} y_{2} \\
& +\mathrm{i} R_{x}\left[\phi_{2}\left(y_{11}-\frac{1}{2} y_{14}\right)+g_{2} y_{2}\right] \text {, } \\
& y_{16}^{\prime}=k\left[-3 y_{16}+y_{19}\right]+f_{1}\left[2 y_{14}+y_{16}\right]+f_{2} y_{12}+F_{R_{y}} y_{3}+\mathrm{i} R_{x}\left[\phi_{1} y_{14}+\phi_{2} y_{12}+g_{2} y_{3}\right] \text {, } \\
& y_{17}^{\prime}=k\left[-3 y_{17}+y_{18}-\frac{1}{2} y_{20}\right] \text {, } \\
& y_{18}^{\prime}=k\left[\frac{3}{2} y_{17}-3 y_{18}+y_{19}\right]+f_{1}\left[y_{11}+y_{18}\right]+F_{R_{y}} y_{5}+\mathrm{i} R_{x}\left[-\frac{1}{2} \phi_{2} y_{17}+g_{1} y_{11}+g_{2} y_{5}\right] \text {, } \\
& y_{19}^{\prime}=k\left[-3 y_{19}+y_{20}\right]+f_{1}\left[y_{12}+2 y_{17}+y_{19}\right]+F_{R_{y}} y_{6}+\mathrm{i} R_{x}\left[\phi_{1} y_{17}+g_{1} y_{12}+g_{2} y_{6}\right] \text {, } \\
& y_{20}^{\prime}=-3 k y_{20}+f_{1}\left[y_{14}+y_{20}\right]-f_{2} y_{17}+F_{R_{y}} y_{8}+\mathrm{i} R_{x}\left[-\phi_{2} y_{17}+g_{1} y_{14}+g_{2} y_{8}\right] \text {. }
\end{aligned}
$$

These equations have to be supplemented with the initial conditions required for their numerical integration from $z=0$ to $z=1$ :

$$
y_{20}(0)=1, \quad y_{i}(0)=0 \quad \text { for } \quad i=1, \ldots, 19 .
$$

In addition, we merely have to satisfy the fairly simple boundary condition

$$
y_{1}(1)=0 \text {. }
$$

What matters here is precisely the replacement of the rather troublesome, symmetric boundary conditions (25) accompanying the original Sturm-Liouville problem by the asymmetric boundary conditions (29) and (30), since these new boundary conditions lend themselves to a standard shooting method: just integrate the system (28) with the initial conditions (29), monitor the resulting value of $y_{1}(1)$, and adjust the system's parameters such that this value becomes zero. 
The price to pay for this simplification is a rather complicated system of equations. But still, the spirit underlying these equations is simple. Since we are not interested in the full spectrum of the eigenvalue problem (14) (or, equivalently, in that of the Sturm-Liouville problem (24, 25) ), but only in that value $R_{c}\{\phi\}$ of $R$ where the lowest eigenvalue associated with some profile $\phi$ passes through zero, we were entitled to set $\lambda=0$ in the derivation of the system (28). We then keep both the profile $\phi$ and the wavevector $\mathbf{k}$ fixed when solving the initial value problem (28, 29), and adjust only the parameter $R$. The smallest value of $R$ where $y_{1}(1)$ vanishes then equals that value of $R$ where the smallest eigenvalue $\lambda$ becomes zero, for this particular $\phi$ and $\mathbf{k}$. We denote this $R$ as

$$
R_{0}\{\phi\}(\mathbf{k})
$$

The desired number $R_{c}\{\phi\}$ then is identified as the global minimum, taken over all wavevectors. Since the wavenumbers $k_{x}$ and $k_{y}$ effectively enter into the system (28) only through their squares $k_{x}^{2}$ and $k_{y}^{2}$, we have

$$
R_{c}\{\phi\}=\min _{k_{x} \geq 0, k_{y}>0}\left\{R_{0}\{\phi\}(\mathbf{k})\right\} .
$$

Thus, the evaluation of the spectral constraint, i.e., the determination of the maximal rescaled Reynolds number $R_{c}\{\phi\}$ up to which a given profile $\phi$ remains an admissible test profile for the variational principle (12), requires some labor. One first has to determine, for that $\phi$ and a representative set of wavevectors $\mathbf{k}$, the first zero of $y_{1}(1)$ that occurs when $R$ is increased from zero to positive numbers. This yields the values (31). In a second step one has to locate the global minimum of all these values, which equals $R_{c}\{\phi\}$.

\section{RESULTS}

Now that we are in a position to evaluate the spectral constraint (14) for an arbitrary profile $\phi(z)$, we have to specify a class of test profiles that is likely to exhaust the variational principle (12, 16). Based on the analysis of the restricted Couette problem, we propose the following class of variational profiles:

$$
\phi(z)= \begin{cases}\frac{1}{2}(1-p)+p z-\frac{1}{2}(1-p)(1-z / \delta)^{n} & \text { for } 0 \leq z \leq \delta \\ \frac{1}{2}(1-p)+p z & \text { for } \delta<z<1-\delta . \\ \frac{1}{2}(1-p)+p z+\frac{1}{2}(1-p)(1-(1-z) / \delta)^{n} & \text { for } 1-\delta \leq z \leq 1\end{cases}
$$

These profiles contain three independent parameters:

i.) the boundary layer thickness $\delta\left(0<\delta \leq \frac{1}{2}\right)$,

ii.) the slope $p$ of the profile in the interior $(0<p \leq 1)$, and

iii.) the polynomial order $n(n=4,5, \ldots)$ of the boundary layer segments.

By construction, each $\phi$ is $n-1$ times continuously differentiable at the matching points $z=\delta$ and $z=1-\delta$. Since the right hand side of the system (28) of differential equations contains derivatives of the profile up to the fourth order, the smallest value of $n$ allowed 
by this system is four. In the case $n=4$ one encounters a discontinuity of the fourth derivative. But as long as this discontinuity remains finite, it poses no problem for the numerical integration. For practical computations we restrict the parameter $n$ to values less than or equal to 1000 .

At this point it needs to be emphasized that the variational parameter $n$ plays an important role for the numerical solution of the variational principle. If this parameter was artificially kept fixed, that is, if one considered the profiles (33) with a constant parameter $n=4$, say, then one would inevitably be confronted with serious divergences which are hard to deal with numerically, and therefore would drastically reduce the maximal attainable Reynolds numbers. The formal reasons for these difficulties lie in the expressions $\phi^{(2)}(z) / \phi^{\prime}(z)$ and $\phi^{(3)}(z) / \phi^{\prime}(z)$, which enter into the system (28) through the auxiliary functions (27). Namely, for high Reynolds numbers the optimized profiles tend to become flat in the interior, so that one has to face small denominators.

Since the solution to this technical problem, achieved with the help of the variational parameter $n$, is of outstanding practical importance, we illustrate it with an example. Let us first note that the parameters $\delta$ and $n$ are related to each other by the profile's slope at $z=0$ (or, equivalently, at $z=1$ ):

$$
\phi^{\prime}(0)=p+\frac{n}{2 \delta}(1-p) .
$$

Secondly, we anticipate the high-Re-scaling behavior of the optimized profiles: we have

$$
\phi^{\prime}(0) \sim \alpha R e \text { and } \phi^{\prime}\left(\frac{1}{2}\right)=p \sim \beta R e^{-1}
$$

with constants $\alpha$ and $\beta$, as will be shown later on (cf. Eqs. (39) and (40)). This implies that the ratio $n /(2 \delta)$ in Eq. (34) necessarily has to increase about linearly with $R e$ when $R e$ becomes large. We now focus on two different parameter combinations: (a) $\delta=0.5$ and $n=1000$, and (b) $\delta=0.002$ and $n=4$. In both cases the ratio $n /(2 \delta)$ takes on the value 1000 , and what matters now is the way the divergences of $\phi^{(2)}(z) / \phi^{\prime}(z)$ and $\phi^{(3)}(z) / \phi^{\prime}(z)$ manifest themselves when the profile slope $p$ approaches zero. Case (a) is actually met in high-Re-solutions of the variational principle when the shape of the profiles' boundary segments is allowed to vary, whereas this shape is artificially kept fixed in case (b). In Figs. 2 and 3 we demonstrate for these two cases the response of $\phi^{(2)}(z) / \phi^{\prime}(z)$ to the variation of $p$ from 0.1 to smaller values, depicting only the relevant intervals of $z$. In case (a) the decrease of $p$ merely entails a shift of the smooth ascend from the (negative) value at $z=0$ to zero. In marked contrast, in case (b) the same decrease of $p$ produces a rather sharp singularity in the vicinity of $z=\delta$. The expression $-\phi^{(3)}(z) / \phi^{\prime}(z)$ shows, qualitatively, the same behavior, but even more pronounced. While the functions shown in Fig. 2 are easy to handle, a singularity like the one that develops in Fig. 3 would soon limit the accessible values of $p$, and thus prevent us from reaching asymptotically high Reynolds numbers.

At this point, an important difference between the full three-dimensional plane Couette problem and the restricted, two-dimensional problem without spanwise degrees of freedom shows up. The cumbersome denominator $\phi^{\prime}$, which does not appear in the restricted case, emerges when one eliminates $v_{x}(z)$ from Eq. (19) in order to derive the Sturm-Liouville equation (24). In this way one arrives at Eq. (23), whereas in the restricted case $\left(k_{y}=0\right)$ the condition (20) of divergence-freeness yields an immediate connection between $v_{x}$ and 
$v_{z}^{\prime}$. Here lies the reason why an asymptotic theory of upper bounds for the full Couette problem, paralleling the one developed for the restricted case in Ref. [22], seems to be out of reach. Nevertheless, the model problem has led to the identification of the test profiles (33) as profiles which will provide the best possible bounds on $c_{\varepsilon}$ in the limit of large Reynolds numbers.

In Fig. 1 we visualize a generic representative of the profile family (33). The profile functional (13), which becomes a function of $\delta, p$, and $n$ when applied to the class (33), now reads

$$
D\{\phi\}=\left[\frac{1}{2} \frac{n^{2}}{(2 n-1) \delta}-1\right](1-p)^{2} \equiv \mathcal{D}(\delta, p, n) .
$$

By constructing the lower envelope of all graphs of the type shown in Fig. 1, that are produced in the $\left(R e, c_{\varepsilon}\right)$-plane by the individual test profiles, we are able to compute the optimal upper bound on $c_{\varepsilon}$ obtainable from the profiles (33) for Reynolds numbers up to $R e \approx 10^{6}$. Moreover, from the optimized profiles we can extract the asymptotic scaling of the profile parameters.

In Fig. 5 we report our findings for the optimized upper bound $\overline{c_{\varepsilon}}(R e)$ on $c_{\varepsilon}(R e)$. Remarkably, this variational bound separates with a sharp kink from the lower bound $\underline{c_{\varepsilon}}(R e)=R e^{-1}$ at the energy stability limit, which is located at

$$
R e_{E S}=82.65014887(1), \quad c_{\varepsilon}\left(R e_{E S}\right)=R e_{E S}^{-1}=0.012099191758(2) .
$$

(The numbers in brackets denote the uncertainty of the last digit.) Up to $R e_{E S}$ both bounds coincide, see Eq. (7). The kink of the upper bound at $R e_{E S}$ is no artifact caused by our particular choice of test profiles (33): even though some numerical improvement of the upper bound $\overline{C_{\varepsilon}}$ is possible in the regime of intermediate $R e$ (see below), one will still obtain a bound that increases for Reynolds numbers slightly above $R e_{E S}$. This behavior is related to the singularity of the balance parameter $a$ at $R e_{E S}$ [20,22], and finds its explanation in a change of the role of the optimal background flow. Below $R e_{E S}$ the optimal background flow coincides with the laminar flow, that is, with a stationary solution to the Navier-Stokes equations, but above $R e_{E S}$ this is no longer the case. Therefore, the deviations $\mathbf{v}(\mathbf{x}, t)$ from the background flow below and above $R e_{E S}$ have a different character. For $R e<R e_{E S}$ these deviations are fluctuations around the physically realized laminar flow. Since they will decay at least exponentially in time [23,24], they yield no contribution to $\varepsilon$. For $R e>R e_{E S}$ the deviations can no longer be regarded as "fluctuations" in the usual sense, and do contribute to $\varepsilon$.

The apparent slight non-smoothness of our bound $\overline{c_{\varepsilon}}$ at $R e \approx 200$, on the other hand, indeed is an artifact; it is caused by the restriction of $n$ to values no less than four. We have found empirically that the component $k_{x}$ of the wavevector minimizing $R_{0}\{\phi\}(\mathbf{k})$ always vanishes. With this knowledge, the system (28) can be simplified: the 20-component complex system is converted into a 20-component real one. As a consequence, the parameter value $n=3$ becomes admissible, which results in a noticeable improvement of the upper bound in the intermediate range $R e_{E S}<R e<700$, as shown in the inset of Fig. 5 .

For Reynolds numbers around 740 the upper bound exhibits a pronounced minimum, and then ascends to the asymptotic value 


$$
\lim _{R e \rightarrow \infty} \overline{c_{\varepsilon}}(R e)=0.01087(1) .
$$

This value lies slightly above - but within the uncertainty span of - Busse's asymptotic result (8). Thus, our variational bound possesses the same asymptotic $R e^{0}$-scaling as the bound provided by the Optimum Theory. In Appendix B we treat the variational principle without taking into account the condition $\nabla \cdot \mathbf{V}=0$ in Eqs. (14). This toy problem provides an illustrative example for our methods, and allows a direct comparison of variational results obtained numerically with the help of the profiles (33) to the corresponding results derived by Howard 8,11] in the framework of the Howard-Busse theory. Our numerical value for the asymptotic bound pertaining to this simplified case, $\lim _{R e \rightarrow \infty} \overline{C_{\varepsilon}}(R e) \approx 0.07071$, differs from Howard's analytical value $9 / 128 \approx 0.07031$ by merely $0.6 \%$, even though our variational profiles are specifically adapted to the full problem, and not to this simplified case.

A key for understanding the overall behavior of the variational upper bound lies in the expressions $R_{0}\{\phi\}(\mathbf{k})$, considered for fixed profile $\phi$ as functions of the two-dimensional wavevector $\mathbf{k}$. We have already mentioned the observation that for each considered test profile the absolute minimum of $R_{0}\{\phi\}(\mathbf{k})$ was attained for $k_{x}=0$. This finding may seem surprising on the first glance, but becomes at least plausible with the help of the following argument: if one neglects the condition $\nabla \cdot \mathbf{V}=0$ in Eq. (14), the minimizing $\mathbf{k}$-vector is the zero-vector, as shown in Appendix B. Taking into account $\nabla \cdot \mathbf{V}=0$ then enforces that the minimizing $\mathbf{k}$ becomes non-zero. But since there is no characteristic length-scale in streamwise direction, the minimizing $k_{x}$ remains zero.

There is a further feature that strongly influences the Re-dependence of the optimized profile parameters, and the scaling behavior of the resulting upper bound $\overline{c_{\varepsilon}}(R e)$ : the single minimizing wavenumber $k_{y}$ that characterizes the variational solution at comparatively low $R e$ bifurcates to a pair of minimizing wavenumbers, $k_{y, 1}$ and $k_{y, 2}$, at the Reynolds number

$$
R e_{B} \approx 460
$$

The variational principle possesses the intriguing property that above $R e_{B}$ both corresponding minimal values of $R_{0}\{\phi\}(\mathbf{k}), R_{1}$ and $R_{2}$, are locked to exactly the same value, i.e., two eigenvalues of the eigenvalue problem (14) pass through zero simultaneously. This degeneracy reflects the fact that, physically speaking, the sensitivity of the variational problem to the behavior of the profiles in the boundary layers separates from the sensitivity to the profiles in the interior. A fairly precise mathematical formulation of this statement can be given for the Couette problem without spanwise degrees of freedom [22].

The consequences of this bifurcation for the upper bound on $c_{\varepsilon}$ are dramatic: its approximate $R e^{-1 / 4}$-dependence that prevails for $R e \leq R e_{B}$ changes to the asymptotic Re $e^{0}$-behavior, while the bound passes through its global minimum

$$
{\overline{c_{\varepsilon} \text { min }}}_{00.00970 \text { at }} \operatorname{Re}_{\min } \approx 740
$$

In order to convey some feeling for the way the variational principle works, we draw $R_{0}\{\phi\}(\mathbf{k})$ as function of both components of $\mathbf{k}$, for the optimized profiles corresponding to the Reynolds numbers $R e_{E S}$ (Fig. 6; cf. Eq. (35)) and $R e_{\min }$ (Fig. 7; cf. Eq. (38)), respectively. Figure 8 depicts the minimizing values of $k_{y}$ that belong to the variational bound displayed in Fig. 5 . Whereas the upper $k_{y}$-branch scales proportionally to $R e$ in the limit of large $R e$, the lower 
branch approaches a constant value. This value is precisely the one that also corresponds to the energy stability limit:

$$
\lim _{R e \rightarrow \infty} \frac{k_{y, 1}}{2 \pi}=\frac{k_{E S}}{2 \pi} \approx 0.4960
$$

This finding is rather unexpected, since we have the simple laminar profile at the energy stability limit, and seemingly entirely different, non-linear profiles with an almost flat interior part in the asymptotic regime. However, the restricted model problem has shown that this interior part of the asymptotic profiles can be related to the laminar profile, so that the asymptotic identity of $k_{y, 1}$ and $k_{E S}$ can be proven analytically by mapping the corresponding eigenvalue problems to each other [22].

It is now of considerable mathematical and physical interest to investigate the Redependence of the optimized profile parameters. We have to keep in mind that the background flow $\mathbf{U}(\mathbf{x})=U \phi(z) \hat{\mathbf{x}}$ is, by construction, not a horizontally-averaged mean flow. On the other hand, the optimal profiles $\phi$ invite comparison with physically realized flow profiles [19], even though such a comparison remains speculative. Fig. 9 shows the metamorphosis of the optimal profiles with increasing Reynolds number. By a close inspection of the data underlying this figure, we can clearly distinguish four different regimes:

i.) For $R e_{E S} \leq R e \leq R e_{1} \approx 160$, a deformation of the laminar profile takes place. Whereas the thickness $\delta$ of the boundary layers remains fixed at 0.5 , and the polynomial order $n$ of the boundary segments remains at the smallest possible value 3 , the profile's slope $p$ in the interior decreases from 1 to almost the limiting value 0 . In this regime the upper bound $\overline{c_{\varepsilon}}$ reaches its maximum and starts to descend.

ii.) The following regime, $R e_{1} \leq R e \leq R e_{2} \approx 670$, is characterized by the development of boundary layers. The parameter $\delta$ decreases to its minimal value of about 0.14 , with $n$ remaining fixed at 3 , while the slope $p$ increases again. In this regime the bifurcation of the minimizing $k_{y}$ occurs, which alters the previous approximate $R e^{-1 / 4}$-behavior of the upper bound.

iii.) In the next regime, $R e_{2} \leq R e \leq R e_{3} \approx 1845$, we observe a dramatic change of the shape of the optimized profile's boundary segments. The parameter $n$ - characterizing the shape of these segments by means of their polynomial order - increases from the smallest possible value $n=3$ to $n=34$, thus steepening the profiles in the immediate vicinity of each boundary, thereby effectively generating new internal boundary layers within the original boundary layers. As a consequence of this structural reorganization of the boundary layers, the entire boundary segments, the extensions of which are quantified by $\delta$, start to reach deeper and deeper into the bulk and finally join again, $\delta$ taking on its maximal value 0.5 . In this regime, the upper bound passes through its minimum, see Eq. (38), and then changes the sign of its curvature.

iv.) For $R e$ above $R e_{3}$, we find simple scaling laws for the optimal profile parameters. These scaling laws give rise to a power-law change of the profile with increasing Re: the slope at the boundary $z=0$ (or at $z=1$ ) is given by

$$
\phi^{\prime}(0) \sim \frac{n}{2 \delta} \sim \alpha R e
$$


while the slope at the midpoint $z=\frac{1}{2}$ becomes

$$
\phi^{\prime}\left(\frac{1}{2}\right)=p \sim \beta R e^{-1}
$$

with constants $\alpha$ and $\beta$. Parallel to this power-law change of the profile, the upper bound on $c_{\varepsilon}$ ascends smoothly to its asymptotic value.

If we had not been forced by practical numerical reasons to constrain the shape parameter $n$ to values less than or equal to 1000 , we would have preserved the asymptotic $R e^{1}$-scaling of $n$, while the parameter $\delta$ would have remained fixed at 0.5 . In our numerics the optimal $n$ becomes 1000 at $R e \approx 51880$, and keeping $n$ fixed at $n=1000$ for even higher $R e$ instead of allowing it to increase further then forces $\delta$ to decrease, as follows from Eq. (39). The ensuing increase of the asymptotic value of the upper bound $\overline{c_{\varepsilon}}$ (compared to the value that would be obtainable if one allowed arbitrarily large $n$ ) is quite small, as can be seen from the fact that $\overline{c_{\varepsilon}}(51880)=0.010832(1)$ differs merely by about $0.3 \%$ from the asymptotic value (36).

\section{CONCLUSIONS}

Figure 10 gives a synopsis of rigorous upper bounds on the dimensionless rate of energy dissipation (6) in plane Couette flow that have been found so far. The topmost three lines indicate results derived with the help of an over-restrictive profile constraint [17,,18, 20] stemming from functional estimates. This sharpened constraint over-satisfies the actual spectral constraint (14) and thus leads to bounds exceeding the asymptotic Busse bound (8) by factors of $8.8,7.9$, or 6.6 , respectively. Only if the spectral constraint is properly implemented and evaluated, as done in this work, one can obtain a rigorous bound that practically coincides with the Busse bound in the asymptotic regime, thereby confirming both Busse's pioneering work [9,10] and Kerswell's recent analysis [19].

However, it must be clearly recognized that much more has been achieved than the confirmation of a 25-year old difficult result. Now a variational principle is available that can not only produce asymptotic bounds of high quality, but yields rigorous bounds of the same quality in the entire range from low to asymptotically high Reynolds numbers. For the plane Couette system discussed in this paper, the bound shows a remarkable structure, notably a pronounced minimum followed by a Re-range between 1000 and 1800 in which the bound's curvature changes its sign. This occurs at about those Reynolds numbers where typical laboratory shear flows start to become turbulent. It is tempting to speculate about this coincidence, but such considerations are beyond the scope of the present work.

Of considerable interest is the mechanism that determines the variational upper bound. As depicted in Fig. 8, the minimizing wavenumber $k_{y}$ bifurcates at $R e_{B} \approx 460$, giving rise to one minimizing $k_{y}$-branch that asymptotically approaches a constant, and to another one that scales linearly with $R e$. Since both corresponding minima determine the solutions to the variational principle by taking on the same value, as described in Sec. IV, there are two characteristic lengths that enter into the solution, one scaling with $R e^{0}$, the other with $R e^{-1}$. The analysis of the restricted Couette problem [22] has led to the conclusion that the first, $R e$-independent minimum reflects the response of the variational principle to the optimized 
profiles solely in the interior of the fluid layer, whereas the second minimum reflects the response to solely the profiles' boundary segments. Thus, the two lengths entering the variational solutions can be identified as basically (i) the extension of the interior flat part of the optimized profile, and (ii) the effective width of its boundary segments. It is structural insight of this kind that will be required for the formulation of a more refined variational principle that might ultimately allow one to decide whether there are corrections to classical scaling.

For the moment being, the comparison of our variational bound with the experimental data shown in Fig. 10 demands some modesty. The bound clearly is far from being sharp; the difference between the variational bound $\overline{c_{\varepsilon}}$ and the corresponding data measured by Reichardt [12] for the plane Couette flow or by Lathrop, Fineberg, and Swinney [13] for the small-gap Taylor-Couette system still spans an order of magnitude. In short, one important step has been made, but there is still a long way to go.

Acknowledgements: This work was supported by the Deutsche Forschungsgemeinschaft via the Sonderforschungsbereich "Nichtlineare Dynamik", SFB 185, and by the GermanIsraeli-Foundation (GIF).

\section{APPENDIX A: COMPOUND MATRIX METHOD FOR THE SIXTH-ORDER STURM-LIOUVILLE PROBLEM}

Keeping in mind that we are not interested in the calculation of the entire spectrum furnished by the Sturm-Liouville eigenvalue problem (24) and (25), but in the determination of that value of $R$ where the smallest eigenvalue passes through zero, we can set $\lambda=0$ right from the outset. We thus obtain the new sixth-order Sturm-Liouville problem

$$
\begin{gathered}
v_{z}^{(6)}-2 \frac{\phi^{(2)}}{\phi^{\prime}} v_{z}^{(5)}-\left\{3 k^{2}+\frac{\phi^{(3)}}{\phi^{\prime}}-2\left(\frac{\phi^{(2)}}{\phi^{\prime}}\right)^{2}\right\} v_{z}^{(4)}+\left\{4 k^{2} \frac{\phi^{(2)}}{\phi^{\prime}}+\mathrm{i} k_{x} R \phi^{\prime}\right\} v_{z}^{(3)} \\
+\left\{3 k^{4}+2 k^{2}\left[\frac{\phi^{(3)}}{\phi^{\prime}}-2\left(\frac{\phi^{(2)}}{\phi^{\prime}}\right)^{2}\right]+\mathrm{i} \frac{k_{x}}{2} R \phi^{(2)}\right\} v_{z}^{(2)} \\
-\left\{2 k^{4} \frac{\phi^{(2)}}{\phi^{\prime}}+\mathrm{i} k_{x} R\left[k^{2} \phi^{\prime}-\phi^{(3)}+\frac{\left(\phi^{(2)}\right)^{2}}{\phi^{\prime}}\right]\right\} v_{z}^{\prime} \\
-\left\{k^{6}+k^{4}\left[\frac{\phi^{(3)}}{\phi^{\prime}}-2\left(\frac{\phi^{(2)}}{\phi^{\prime}}\right)^{2}\right]-\frac{1}{4}\left(k_{y} R \phi^{\prime}\right)^{2}+\right. \\
\left.\mathrm{i} \frac{k_{x}}{2} R\left[k^{2} \phi^{(2)}-\phi^{(4)}+3 \frac{\phi^{(3)} \phi^{(2)}}{\phi^{\prime}}-2 \frac{\left(\phi^{(2)}\right)^{3}}{\left(\phi^{\prime}\right)^{2}}\right]\right\} v_{z}=0,
\end{gathered}
$$

together with the boundary conditions

$$
\left.v_{z}(z)\right|_{z=0,1}=0,\left.\quad v_{z}^{\prime}(z)\right|_{z=0,1}=0, \quad \text { and }\left.\quad\left[v_{z}^{(2)}(z)-\frac{1}{2 k^{2}} v_{z}^{(4)}(z)\right]\right|_{z=0,1}=0
$$


The eigenvalues of this problem are those values of $R$ where both Eqs. (A1) and (A2) are satisfied.

In order to integrate Eq. (A1), one has to pose suitable initial conditions at one of the boundaries, let us say $z=0$. We define the six-vectors

$$
\mathbf{V}_{z}(z) \equiv\left(v_{z}(z), v_{z}^{\prime}(z), v_{z}^{(2)}(z)-\frac{1}{2 k^{2}} v_{z}^{(4)}(z), v_{z}^{(3)}(z), v_{z}^{(4)}(z), v_{z}^{(5)}(z)\right)^{T}
$$

and consider the three linearly independent fundamental solutions $\mathbf{V}_{z, 1}(z), \mathbf{V}_{z, 2}(z)$, and $\mathbf{V}_{z, 3}(z)$ to (A1) that emerge from the initial values

$\mathbf{V}_{z, 1}(0)=(0,0,0,1,0,0)^{T}, \quad \mathbf{V}_{z, 2}(0)=(0,0,0,0,1,0)^{T}, \quad$ and $\quad \mathbf{V}_{z, 3}(0)=(0,0,0,0,0,1)^{T}$

respectively. By construction, each of these three solutions satisfies the conditions (A2) at $z=0$, and the most general solution obeying these one-sided b.c. is just a superposition of them. That solution which also satisfies the conditions (A2) at the other boundary $z=1$ is then singled out by the requirement

$$
\operatorname{Det}\left(\begin{array}{ccc}
v_{z, 1}(1) & v_{z, 2}(1) & v_{z, 3}(1) \\
v_{z, 1}^{\prime}(1) & v_{z, 2}^{\prime}(1) & v_{z, 3}^{\prime}(1) \\
v_{z, 1}^{(2)}(1)-\frac{1}{2 k^{2}} v_{z, 1}^{(4)}(1) & v_{z, 2}^{(2)}(1)-\frac{1}{2 k^{2}} v_{z, 2}^{(4)}(1) & v_{z, 3}^{(2)}(1)-\frac{1}{2 k^{2}} v_{z, 3}^{(4)}(1)
\end{array}\right)=0
$$

But from the numerical point of view the evaluation of this determinant, which in most cases necessitates to subtract large numbers of almost equal magnitude from each other, is rather inconvenient. An efficient and numerically stable way to bypass this difficulty has become known as compound matrix method in the literature, see, e.g., Ref. [27 for an introduction to the method, or Ref. 222] for applications to the restricted Couette problem. For applying this technique to the full Couette problem studied in this paper, we define a new vector $\mathbf{y}(z)=\left(y_{1}(z), \ldots, y_{20}(z)\right)^{T}$, the 20 components of which are proportional to the $3 \times 3$ minors of the $6 \times 3$ solution matrix that contains $\mathbf{V}_{z, 1}$ as its first, $\mathbf{V}_{z, 2}$ as its second, and $\mathbf{V}_{z, 3}$ as its third column:

$$
\begin{aligned}
y_{1} & \equiv k^{9} \sum_{\sigma} \operatorname{sign}(\sigma) v_{z, \sigma(1)} v_{z, \sigma(2)}^{\prime}\left(v_{z, \sigma(3)}^{(2)}-\frac{1}{2 k^{2}} v_{z, \sigma(3)}^{(4)}\right), \\
& \vdots \\
y_{20} & \equiv \sum_{\sigma} \operatorname{sign}(\sigma) v_{z, \sigma(1)}^{(3)} v_{z, \sigma(2)}^{(4)} v_{z, \sigma(3)}^{(5)} .
\end{aligned}
$$

The summations involve all permutations $\sigma$ of $1,2,3$. The powers of $k$ multiplying each sum are determined in the following way: the expression defining $y_{20}$ carries the highest total number of derivatives, namely 12 ; this expression is multiplied by $k^{0}$. Descending in the index from 20 to 1 , the number of derivatives is successively diminished; each derivative less gives a factor of $k$ more. This guarantees two things: firstly, the initial conditions (29) are independent of $k$, and secondly, all components have the same order of magnitude.

From Eq. (A1) one then obtains a system of first-order equations that already closely resembles the system (28) which we have studied numerically, with the only difference that 
in each component $i(i=1, \ldots, 20)$ the term $-3 k y_{i}$ appearing in (28) is still missing. It is obvious that the initial conditions (29) and the boundary condition (30) follow directly from (A3) and the definitions (A4).

This term $-3 k y_{i}$ in the $i$-th component of the system is of particular importance. The restricted Couette problem has taught us the lesson [22] that the system of first-order equations directly provided by the compound matrix method is not suited for numerical analysis in the high-Re-regime, where one has to monitor large values of $k$. One rather has to rescale each component of the system by a common exponential damping factor. The reason for this rescaling is related to the fact that the optimized profiles tend to become flat in the interior of the fluid layer when Re becomes high, while the extension of the boundary segments, where the profiles have to bend in order to meet the boundary conditions $\phi(0)=0$ and $\phi(1)=1$, tends to zero. This motivates to neglect the profiles' boundary conditions altogether, and to consider Eq. (A1) with a linear profile $\phi(z)=c z$, so that $\phi^{\prime}$ becomes equal to the constant $c$, and all higher profile derivatives vanish. In the limit $c \rightarrow 0$ one then arrives at

$$
v_{z}^{(6)}-3 k^{2} v_{z}^{(4)}+3 k^{4} v_{z}^{(2)}-k^{6} v_{z}=0,
$$

with the b.c. (A2). Applying the compound matrix technique as sketched above to this boundary value problem, one finds a 20-component system of first-order equations as in the case of a general $\phi$, but now with constant coefficients. This system can be solved analytically by standard means, but an accurate numerical solution for large $k$ 's will be possible only if the solutions do not grow (or decrease) exponentially. Thus, the intended numerical approach forces us to rescale the system such that the largest eigenvalue of the rescaled system's coefficient matrix is exactly equal to zero. It turns out to be possible to determine the Jordan normal form of the $20 \times 20$-matrix resulting from Eq. (A5) analytically; the largest eigenvalue is non-degenerate and equals $3 k$. Hence, the transformation

$$
\tilde{y}_{1}(z) \equiv y_{1}(z) \mathrm{e}^{-3 k z}, \quad \cdots \quad, \quad \tilde{y}_{20}(z) \equiv y_{20}(z) \mathrm{e}^{-3 k z},
$$

has the desired effect. Applying the very same transformation also to the system given by the compound matrix method for the case of a general candidate profile $\phi$ (and finally omitting the tilde-signs), changes that system merely be adding $-3 k y_{i}$ to the $i$-th component, and thus produces our system (28). We emphasize that the identification of the proper exponential scaling factor, and the actual scaling transformation (A6), is crucial for obtaining equations that remain numerically stable even in the regime of asymptotically high Reynolds numbers.

A striking feature found in the study of the restricted Couette problem, where the system corresponding to (28) consists of merely six equations [22], is the possibility to reduce that complex 6-component system to a real 6-component system, thereby halving the number of equations. This simplification could be obtained from taking $\operatorname{Im}\left(y_{1}(z)\right)=0$, for all $z$. Even though we have strong numerical evidence that the imaginary part of the component $y_{1}(z)$ vanishes also in the present case, we did not succeed in deriving a reduced system for arbitrary $\phi$. Hence, we make a virtue of necessity and keep the full system (28), but exploit our insight for controlling the accuracy of the numerical solutions by monitoring the magnitude of $\operatorname{Im}\left(y_{1}(z)\right)$. 


\section{APPENDIX B: NEGLECTING $\nabla \cdot \mathrm{V}=0$}

In this appendix we study the variational problem for energy dissipation in plane Couette flow without requiring that the solutions $\mathbf{V}(\mathbf{x})$ to the eigenvalue problem (14) be divergencefree. Then the solutions to this eigenvalue problem are determined in an enlarged space of functions, so that the bound obtainable for this simplified problem can naturally not be as good as the one reported in Sec. IV. However, the distinct value of this toy model lies in two points. Firstly, we can illustrate, condensed in a nutshell, the principles of both the background flow method itself and the use of the compound matrix technique. Secondly, this model allows an immediate quantitative comparison of our numerically calculated, asymptotic dissipation bound with the corresponding value provided by the Howard-Busse theory.

To begin with, the Eqs. (17)-(20) for the spectral constraint's eigenvalue problem simplify enormously when the condition $\nabla \cdot \mathbf{V}=0$ is skipped:

$$
\begin{aligned}
& \lambda v_{x}=-2\left(\partial_{z}^{2}-k^{2}\right) v_{x}+R \phi^{\prime} v_{z}, \\
& \lambda v_{y}=-2\left(\partial_{z}^{2}-k^{2}\right) v_{y}, \\
& \lambda v_{z}=-2\left(\partial_{z}^{2}-k^{2}\right) v_{z}+R \phi^{\prime} v_{x} .
\end{aligned}
$$

Equation (B2) separates from the others, and we immediately obtain a solution that is compatible with the b.c. (21) for each $\lambda$ and $k$, namely $v_{y}(z)=0$. Introducing the new functions $v(z) \equiv v_{x}(z)-v_{z}(z)$ and $w(z) \equiv v_{x}(z)+v_{z}(z)$, Eqs. (B1) and (B3) transform into

$$
\begin{aligned}
& \lambda v=-2\left(\partial_{z}^{2}-k^{2}\right) v-R \phi^{\prime} v \\
& \lambda w=-2\left(\partial_{z}^{2}-k^{2}\right) w+R \phi^{\prime} w
\end{aligned}
$$

the b.c. $\operatorname{read} v(0)=w(0)=0$ and $v(1)=w(1)=0$.

We now assume that the profile functions $\phi(z)$ satisfy, besides the conditions posed in Eq. (11), also the additional monotony condition

$$
\phi^{\prime}(z) \geq 0 \text { for } 0 \leq z \leq 1
$$

Our test profiles (33) obviously comply with this plausible requirement. Hence, if the com-

ponent $w(z)$ does not vanish identically, the positive-definiteness of $-\partial_{z}^{2}$ will enforce $\lambda>0$ for positive $R$. Consequently, for $\lambda=0$ and $R>0$ we have $w(z)=0$, and are left with the second-order Sturm-Liouville boundary value problem

$$
v^{\prime \prime}-\left(k^{2}-\frac{1}{2} R \phi^{\prime}\right) v=0, \quad v(0)=v(1)=0 .
$$

In exact analogy to the full problem, the main task posed by the spectral constraint is to calculate for given $\phi$ and $k \geq 0$ the smallest $R$-value $R\{\phi\}(k)$ where (B5) is fulfilled, and then to minimize over all $k$ in order to extract $R_{c}\{\phi\}$. But now the second-order differential equation (B5) shows that the minimizing $k$-value $k_{c}\{\phi\}$ equals zero for each $\phi(z)$. In the final step, the optimal upper bound on $c_{\varepsilon}(R e)$ follows from inequality (16).

The laminar profile $\phi(z)=z$ yields the "energy stability limit" for our toy problem,

$$
R e_{E S}=2 \pi^{2} \approx 19.74, \quad c_{\varepsilon}\left(R e_{E S}\right)=1 / 2 \pi^{2} \approx 0.05066
$$


In order to compute $R_{c}\{\phi\}$ for non-laminar profiles we now rewrite the second-order equation $(\mathbb{B 5})$ as a system of first-order equations by defining the vector $\mathbf{y}(z) \equiv\left(v(z), v^{\prime}(z)\right)^{T}$. In this way we get

$$
y_{1}^{\prime}=y_{2}, \quad y_{2}^{\prime}=-\frac{1}{2} R \phi^{\prime} y_{1},
$$

where we have set $k=0$; initial conditions at $z=0$ and b.c. at $z=1$ are given by

$$
\mathbf{y}(0)=(0,1)^{T}, \quad y_{1}(1)=0
$$

This reformulation of the Sturm-Liouville problem (B5) corresponds precisely to the reformulation provided by the compound matrix method. If one compares this simple boundary value problem (B6, B7) to the corresponding boundary value problem (28)-(30), one gets a vivid impression of the complications introduced into the variational principle by the condition $\nabla \cdot \mathbf{V}=0$.

Figure 11 shows the numerically computed variational upper bound on $c_{\varepsilon}(R e)$ that results from inequality (12) when $\nabla \cdot \mathbf{V}=0$ is neglected, i.e., when the spectral constraint is translated into Eqs. (B6, B7), and the previous test profiles (33) are employed. For simplicity, we have fixed the parameter $n$ to the value $n=2$. Interestingly, this bound exhibits the same shape as the one that had been found analytically [20] when another overrestrictive profile constraint stemming from functional estimates had been used instead of the actual spectral constraint; that bound is indicated by the long-dashed line in figure 10. The best possible asymptotic upper bound $\overline{c_{\varepsilon}}(R e)$ for the toy model without $\nabla \cdot \mathbf{V}=0$ can be found for a non-integer value of $n$, namely $n_{\infty} \approx 3.40$ :

$$
\lim _{R e \rightarrow \infty} \overline{c_{\varepsilon}}(R e) \approx 0.07071
$$

This value has to be compared to the bound calculated analytically by Howard [8,11], also without accounting for divergence-freeness:

$$
\lim _{R e \rightarrow \infty} \overline{C_{\varepsilon}}(R e)=\frac{9}{128} \approx 0.07031
$$

The work of Kerswell [19] suggests that both the Howard-Busse theory and the background flow method, when fully exhausted, give the same asymptotic upper bound. Even though we have deliberately used test profiles which are not specifically adapted to the present toy model, we have missed Howard's bound by merely $0.57 \%$. 


\section{REFERENCES}

[1] A.N. Kolmogorov, C. R. Acad. Nauk USSR 30, 301 (1941).

[2] A.M. Obukhov, C. R. Acad. Nauk USSR 32, 22 (1941).

[3] W. Heisenberg, Z. Phys. 124628 (1948).

[4] C.F. von Weizsäcker, Z. Phys. 124, 614 (1948).

[5] L. Onsager, Phys. Rev. 68, 285 (1945).

[6] S. Grossmann, Phys. Rev. E 51, 6275 (1995).

[7] G. Stolovitzky and K.R. Sreenivasan, Phys. Rev. E 52, 3242 (1995).

[8] L.N. Howard, Ann. Rev. Fluid Mech. 4, 473 (1972).

[9] F.H. Busse, J. Fluid Mech. 41, 219 (1970).

[10] F.H. Busse, Adv. Appl. Mech. 18, 77 (1978).

[11] F.H. Busse, in: Nonlinear Physics of Complex Systems - Current Status and Future Trends, J. Parisi, S.C. Müller, and W. Zimmermann, eds., Lecture Notes in Physics, vol. 476, 1 (Springer-Verlag, Berlin, 1996).

[12] H. Reichardt, Gesetzmäßigkeiten der geradlinigen turbulenten Couetteströmung. Mitteilungen aus dem Max-Planck-Institut für Strömungsforschung, Göttingen, No. 22 (1959).

[13] D.P. Lathrop, J. Fineberg and H.L. Swinney, Phys. Rev. Lett. 68, 1515 (1992).

[14] C.R. Doering and P. Constantin, Phys. Rev. E 49, 4087 (1994).

[15] P. Constantin and C.R. Doering, Phys. Rev. E 51, 3192 (1995).

[16] C.R. Doering and P. Constantin, Phys. Rev. E 53, 5957 (1996).

[17] C.R. Doering and P. Constantin, Phys. Rev. Lett. 69, 1648 (1992).

[18] T. Gebhardt, S. Grossmann, M. Holthaus, and M. Löhden, Phys. Rev. E 51, 360 (1995).

[19] R.R. Kerswell, Physica D 100, 355 (1997).

[20] R. Nicodemus, S. Grossmann and M. Holthaus, Physica D 101, 178 (1997).

[21] C.R. Doering and J.M. Hyman, Phys. Rev. E 55, 7775 (1997).

[22] R. Nicodemus, S. Grossmann and M. Holthaus, The background flow method. Part 1: Constructive approach to bounds on energy dissipation. Part 2: Asymptotic theory of dissipation bounds. Preprints (1997).

[23] D.D. Joseph, Stability of Fluid Motions I $\mathscr{E}$ II (Springer-Verlag, Berlin, 1976).

[24] P.G. Drazin and W.H. Reid, Hydrodynamic Stability (Cambridge University Press, Cambridge, 1981).

[25] As as result of various approximations used in its derivation, the numerical value of the bound (8) is afflicted with some uncertainty, which is possibly of the order of $20 \%$. (F.H. Busse, private communication, 1996).

[26] E. Hopf, Math. Annalen 117, 764 (1941).

[27] B. Straughan, The energy method, stability, and nonlinear convection (Springer-Verlag, New York, 1992). 


\section{FIGURES}

FIG. 1. Graph of an upper bound on $c_{\varepsilon}$ produced according to Eq. (16) by the profile $\phi$ shown in the inset.

FIG. 2. Behavior of $\phi^{(2)}(z) / \phi^{\prime}(z)$ in the interval $0 \leq z \leq 0.015$ for $\delta=0.5$ and $n=1000$. From left to right: graphs that result when $p$ is successively diminished by factors of $1 / 10$ from $p=0.1$ to $p=10^{-6}$.

FIG. 3. Behavior of $\phi^{(2)}(z) / \phi^{\prime}(z)$ in the vicinity of $z=\delta$ for $\delta=0.002$ and $n=4$. From top to bottom: graphs that result when $p$ is successively diminished by factors of $1 / 10$ from $p=0.1$ to $p=10^{-6}$, cf. Fig. 2.

FIG. 4. Test profile $\phi(z)$ as given by Eq. (33), for a generic parameter set $(n=4, \delta=0.3$, $p=0.1$.

FIG. 5. Bounds on $c_{\varepsilon}$ for the plane Couette flow. Points denote the variational upper bound $\overline{c_{\varepsilon}}(R e)$ computed numerically with the test profiles $(\overline{33})$; the solid line on the left is the lower bound $\underline{c_{\varepsilon}}(R e)=R e^{-1}$. The asymptotic value of the upper bound, $\lim _{R e \rightarrow \infty} \overline{c_{\varepsilon}}(R e)=0.01087(1)$, lies slightly above, but within the uncertainty span of Busse's asymptotic result (8). - The inset depicts the improvement obtained by preassuming that the wavevector minimizing $R_{0}\{\phi\}(\mathbf{k})$ has a vanishing component in streamwise direction. Solid line: lower bound $\underline{c_{\varepsilon}}(R e)$; dashed line: same upper bound as in the main figure; points: improvement of the bound obtained by admitting the parameter value $n=3$.

FIG. 6. Three-dimensional surface plot visualizing the k-dependence of $R_{0}\{\phi\}(\mathbf{k})$ for the laminar profile, which is the optimized profile for Reynolds numbers up to the energy stability limit $R e_{E S}$.

FIG. 7. Three-dimensional surface plot visualizing the k-dependence of $R_{0}\{\phi\}(\mathbf{k})$ for the optimized profile belonging to the absolute minimum of the upper bound on $c_{\varepsilon}$ at $R e=R e_{\min } \approx 740$. Note the appearance of two distinct minima on the $k_{y}$-axis. Since the profile results from the variational principle, both minima have the same value.

FIG. 8. Minimizing wavenumber(s) $k_{y}$ corresponding to the upper bound on $c_{\varepsilon}$ displayed in Fig. 5 .

FIG. 9. Three-dimensional plot visualizing the metamorphosis of the optimized variational profiles with the increase of the Reynolds number. We have depicted the most important Re-interval on a logarithmic scale, beginning with the energy stability limit $R e_{E S}$ and ending in the scaling regime $R e>R e_{3}$. 
FIG. 10. Comparison of bounds on $c_{\varepsilon}(R e)$ for the plane Couette flow. - Solid slanted straight line: lower bound $\underline{c_{\varepsilon}}(R e)=R e^{-1}$. - Topmost horizontal solid line: upper bound obtained by Doering and Constantin in Refs. [14, 17] with the help of an over-restrictive profile constraint and piecewise linear profiles; $\overline{c_{\varepsilon}}(R e) \approx 0.088$ for $R e>11.32$. - Dashed-dotted line: improved bound on $c_{\varepsilon}(R e)$ derived by Gebhardt et al. in Ref. [18] from the analytical solution of the Doering-Constantin principle with the over-restrictive constraint; $\overline{c_{\varepsilon}}(R e) \approx 0.079$ for $R e>16.98$. - Long-dashed line: further improvement due to the introduction of the balance parameter in Ref. [20]; $\overline{c_{\varepsilon}}(R e) \rightarrow 0.066$. This bound is still calculated analytically utilizing the over-restrictive profile constraint. - Heavy dots: upper bound for the plane Couette flow obtained in this work from the variational principle (12) with the actual spectral constraint (14), cf. Fig. 5; $\overline{c_{\varepsilon}}(R e) \rightarrow 0.01087(1)$. The variational profiles are given by Eq. (33), including $n=3$ as an admissible parameter. - Joining dashed line: asymptotic upper bound (8) derived by Busse in Refs. [9, 10]; $\overline{c_{\varepsilon}}(R e) \rightarrow 0.010(1)$. The shaded area denotes the estimated uncertainty of this bound. - Triangles: experimental dissipation rates for the plane Couette flow measured by Reichardt [12]. - Circles: experimental dissipation rates for the Taylor-Couette system with small gap as measured by Lathrop, Fineberg and Swinney [13].

FIG. 11. Bounds on $c_{\varepsilon}(R e)$ for the plane Couette flow obtained by neglecting the condition $\nabla \cdot \mathbf{V}=0$ in the eigenvalue equations (14). Points denote the variational upper bound $\overline{c_{\varepsilon}}(R e)$, computed numerically from the test profiles (33) with fixed parameter $n=2$; the solid line on the left is the lower bound $\underline{c_{\varepsilon}}(R e)=R e^{-1}$. 